BMJ Open

Sport \&

Exercise

Medicine

\title{
Barbell back squat: how do resistance bands affect muscle activation and knee kinematics?
}

\author{
Madeleine B Reece, ${ }^{1}$ Graham P Arnold, ${ }^{1}$ Sadiq Nasir, ${ }^{1}$ Weijie W Wang, \\ Rami Abboud (1D ${ }^{2}$
}

To cite: Reece MB, Arnold GP, Nasir S, et al. Barbell back squat: how do resistance bands affect muscle activation and knee kinematics? BMJ Open Sport \& Exercise Medicine 2020;6:e000610. doi:10.1136/ bmjsem-2019-000610

Accepted 8 January 2020

Check for updates

C) Author(s) (or their employer(s)) 2020. Re-use permitted under CC BY-NC. No commercial re-use. See rights and permissions. Published by BMJ.

${ }^{1}$ nstitute of Motion Analysis \& Research (IMAR), University of Dundee, Dundee, UK ${ }^{2}$ Dean's Office, University of Balamand Faculty of Engineering, El-Koura, Lebanon

Correspondence to Dr Graham P Arnold; g.p.arnold@dundee.ac.uk

Professor Rami Abboud; rjabboud@balamand.edu.Ib

\section{ABSTRACT}

Objectives This study aimed to determine whether looped resistance bands affect knee kinematics and lower body muscle activation during the barbell back squat. Methods Twenty-six healthy participants (13 female, 13 male) calculated their one repetition maximum (RM) prior to data collection. Each participant performed three squats at both $80 \%$ and $40 \% 1 \mathrm{RM}$ wearing a light resistance band, an extra-heavy resistance band and no resistance band.

Vicon 3D motion analysis cameras were used to collect the kinematic data, and Delsys Trigno Lab wireless electromyography (EMG) system was used to measure vastus medialis, vastus lateralis, gluteus maximus, gluteus medius and biceps femoris muscle activity. Peak knee flexion angle, peak knee valgus angle and maximum tibial rotation values were examined. Peak EMG values were also analysed after being normalised and expressed as a percentage of maximum voluntary contraction (MVC). Results Gluteus maximus (GM) activity is significantly increased when a resistance band is used during squatting. However, squatting with a resistance band is detrimental to knee kinematics as it leads to an increase in knee valgus angle and maximum tibial rotation angle. A direct correlation is recorded between an increase in resistance and an increase in these two angles.

Conclusions Squatting with resistance bands is likely to increase the risk of knee injury. Coaches and clinicians who already implement this technique are advised to remove resistance band squats from training and rehabilitation programmes. Further research evaluating the long-term effects of using resistance bands during the barbell back squat should be considered.

\section{INTRODUCTION}

The barbell back squat is a strengthening training exercise that is continuing to grow in popularity. As a compound exercise, it develops the most powerful muscles in the human body. The exercise attracts both professional athletes and recreational gym goers as well as playing an important role in rehabilitation situations. ${ }^{12}$ As the feet remain in a fixed position throughout the squat movement, the exercise is classified as a closed kinetic chain exercise. It is often
Summary box

Looped resistance bands placed around the distal thigh during the barbell back squat:

- increases gluteus maximus muscle activation,

- increases peak knee valgus angle and maximum tibial rotation angle.

prescribed with regard to the rehabilitation of anterior cruciate ligament (ACL) injuries, as the forces on the ACL are lower than other exercises such as knee extension. ${ }^{2}$

If the squat is not performed in alignment with the recommended technique, the risk of injury occurring increases. Movement of the knee in the frontal plane is one factor that increases the risk of injury sustained to the knee when squatting. ${ }^{3}$ Medial knee displacement (MKD) is particularly abundant among inexperienced squatters and is described as the movement of the knees towards the midline, resulting in a knee position medial to the great toe (figure 1). ${ }^{4-7}$ It is thought to originate from an inability of the hip muscles to support the femur resulting in excessive adduction and internal rotation. ${ }^{4}$ The ACL directly resists internal tibial rotation as well as opposing knee valgus, making this structure susceptible to injury if MKD occurs during squatting. ${ }^{2}$

The use of a looped resistance band as a proprioceptive aid when squatting is a technique already implemented by clinicians and coaches to maintain a neutral knee alignment. Despite this, recent studies have disagreed that resistance bands are capable of achieving this result. ${ }^{38}$ However, it has been shown that resistance bands can increase the activation of certain lower limb muscles during the movement, which may work to prevent knee valgus. ${ }^{9}$ The focus in many of these studies was not on weighted squats alone, but rather on a combination of bodyweight squats and/ or jumping squats, rendering the results less relevant to athletes and gym goers. Only one 

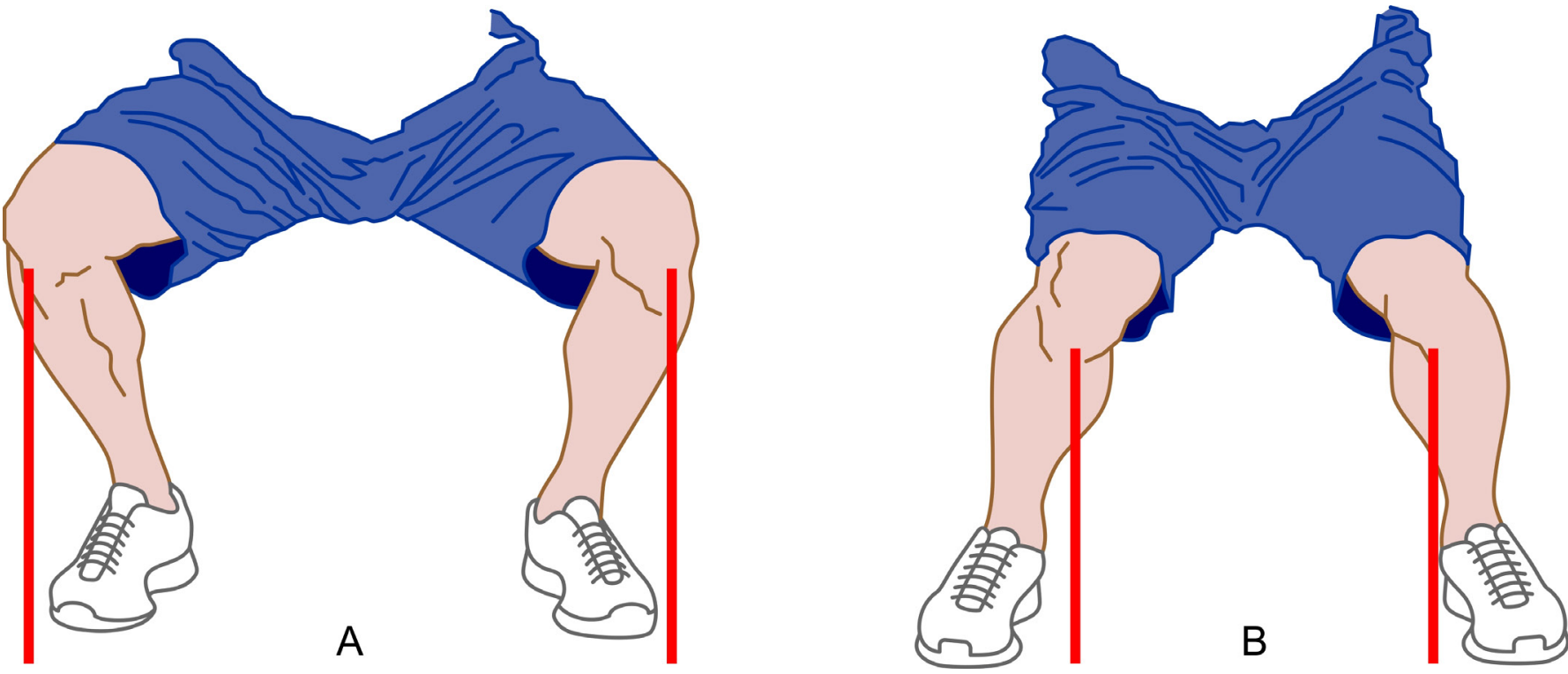

Figure 1 Normal knee alignment in the squat $(A)$ and valgus knee alignment in the squat (B).

study, conducted by Foley et al (2017), investigated both EMG amplitudes of lower limb muscles and medial knee collapse during a banded barbell back squat. Their paper was methodologically limited as neither the internal tibial rotation nor knee valgus angles were calculated. ${ }^{8}$ Foley $e t$ al study also excluded women, despite ACL injuries in women being 4-6 times higher than their male counterparts. ${ }^{10}$

Therefore, the primary aim of this study was to supply more conclusive evidence to support whether looped resistance bands reduce peak knee valgus and internal tibial rotation and therefore ACL strain. A secondary aim was to investigate the effect resistance bands have on the muscle activation of vastus lateralis (VL), vastus medialis (VM), biceps femoris (BF), gluteus medius and gluteus maximus (GM) during the barbell squat.

\section{METHODS}

\section{Study design}

A repeated measures design was employed to examine the effect of using resistance bands on frontal plane knee mechanics and lower limb muscle activation during the barbell back squat.

\section{Patient and public involvement}

A convenience sample of 26 healthy (13 male, 13 female) participants volunteered for the study. This is the largest number of participants of any study in this field to date and the only study to investigate females using resistance bands during the barbell back squat to our knowledge. Participant requirements included: at least 6 months experience of squatting, novice user of looped resistance bands, over the age of 18 and be in good general health at the time of the study. The mean height, mass and age of participants was $172.3 \mathrm{~cm} \pm 6.9,73.3 \mathrm{~kg} \pm 9.6$ and 21.7 years \pm 1.3 , respectively. Participants were verbally informed of all procedures and signed a written consent form. Ethical approval was gained from the University Medical Research Ethics Committee. No patients were involved in this study.

\section{Instrumentation}

The collection of motion data was synchronous with EMG data.

Delsys Trigno Lab wireless EMG system was used for data collection of muscle activity. Nine $37 \mathrm{~mm} \times 26 \mathrm{~mm} \times$ $15 \mathrm{~mm}$ EMG sensors were attached, after hair removal and skin cleaning with NuPrep gel, over the muscle bellies of VM, VL, BF, GM bilaterally and the right gluteus medius. EMG data were captured at $1000 \mathrm{~Hz}$ with the bandwidth of $10-850 \mathrm{~Hz}$ and a CMRR of $<-80 \mathrm{~dB}$.

The Vicon Nexus system V.2.7.1 was used for collection of motion analysis data. Fourteen MXF40 cameras were used, each recording at $200 \mathrm{~Hz}$ at 4 megapixel resolution. Twenty $14 \mathrm{~mm}$ diameter, retroreflective markers were placed on each participant in alignment with the plug-in gait model (figure 2).

Two resistance bands were tested, the light and extra heavy Corezone resistance bands. The elastic rate of the light band was $0.21 \mathrm{~N} / \mathrm{mm}$, whereas the elastic rate of the extra heavy band was $0.68 \mathrm{~N} / \mathrm{mm}^{11}$

\section{Procedures}

All data were collected during one single session at IMAR's gait laboratory. Before attending the session, participants were asked to determine their $1 \mathrm{RM}$.

First, the height $(\mathrm{cm})$, weight $(\mathrm{kg})$, knee width $(\mathrm{mm})$, ankle width $(\mathrm{mm})$, inter-ASIS distance $(\mathrm{mm})$ and leg length $(\mathrm{mm})$ of each participant were recorded. Participants warmed up in whichever way they felt most comfortable, in order to accommodate the different levels of ability between participants. Retro-reflective markers and EMG sensors were attached to the relevant locations on the body and a standard $\mathrm{T}$ frame was used 


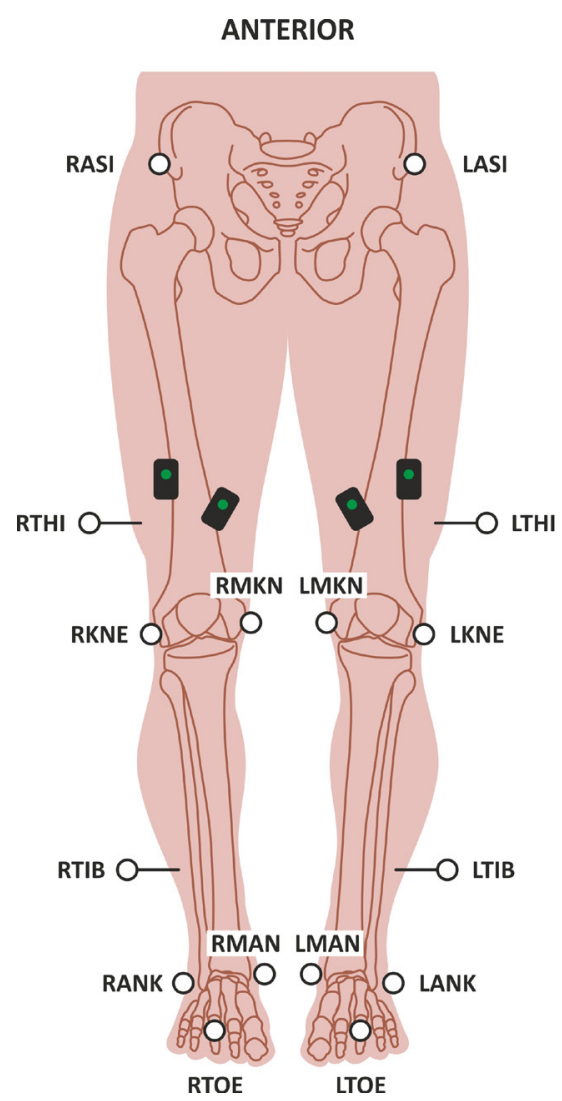

LATERAL

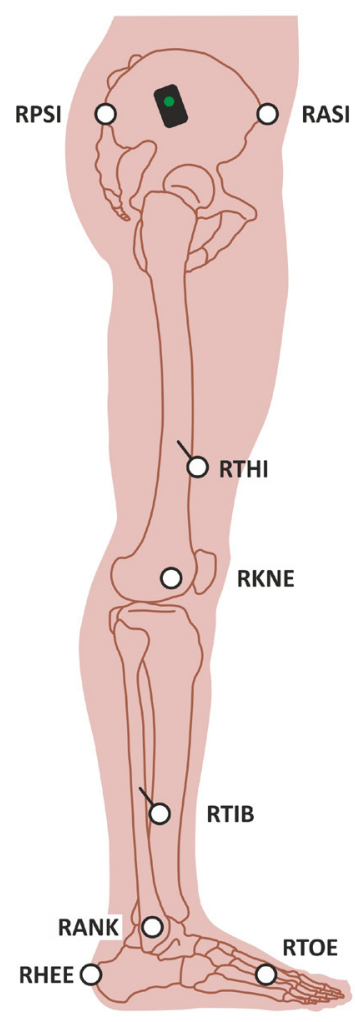

POSTERIOR

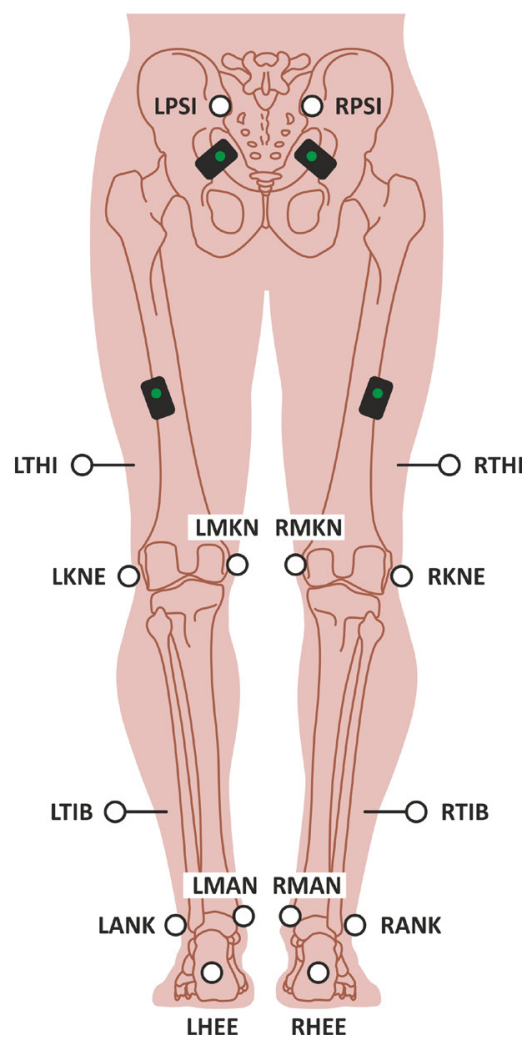

Figure 2 Retroreflective marker and EMG sensor placement.

to statically and dynamically calibrate the Vicon Nexus system.

The MVC value of each muscle tested was required for normalisation of the data. The dynamic normalisation task used was recommend by Balshaw and Hunter (2012). ${ }^{12}$ First, the participant's 3RM was calculated, by multiplying their 1RM by $0.92,{ }^{13}$ which was summed with $88.6 \%$ of their body mass, resulting in their 3RM system mass. The normalisation task required $80 \%$ of this load to be squatted five times. Peak muscle activity over the five repetitions for each muscle was taken as the MVC value.

Before data collection, the Vicon 3D motion system required further static and dynamic calibration which involved the participant standing in a T-pose for three seconds and then walking up and down the lab. During data collection, each participant completed three repetitions of the barbell back squat, lifting 80\% 1RM and $40 \%$ 1RM (separately), under the following conditions: no band, light band and extra heavy band. The order of completion was randomised. A number of factors were controlled: squat width was set to be slightly wider than shoulder width, all participants squatted barefoot and adopted a high bar placement, resistance bands were placed just above the lateral epicondyle of the femur, speed was standardised at $5 \mathrm{~s}$ to complete the full movement. A $5 \mathrm{~min}$ rest period was provided between each set and participants were continually instructed to push their knees out as much as possible against the resistance of the band in order to maintain knee alignment over the great toe throughout the movement.

\section{Data analysis}

Each trial was labelled using Vicon software and three events were added: the start of the squat, the deepest point of the squat and the end point of the ascent phase. The deepest point of the squat was identified as the point of greatest vertical displacement of the ASIS markers. Once labelled, a CSV file from the Nexus software for each trial was uploaded to a custom written extraction programme, producing an Excel file which indicated the peak knee angles during both the concentric (ascent) and eccentric (descent) phases of the squat.

EMG data for each muscle from each trial were exported from Nexus as CVS files and was uploaded to the Nexus 2 EMG analyser programme, producing an Excel file which indicated (as a \% of the MVC) the peak EMG muscle contraction for each muscle investigated during the eccentric and concentric phases of the squat.

\section{Statistical analysis}

Statistical analyses were carried out using Statistical Package for Social Sciences (SPSS) V.22.0. Statistical significance was determined at $\mathrm{p}<0.05$.

Kinematic and EMG data were analysed using the General Linear Model Repeated Measures to calculate means and SE of means. Each subject has three trials per condition and the number of trials is balanced for all subjects; therefore, 
we did not consider that we needed corrections. Means, SE of means and $p$ values were taken from estimates and pairwise comparisons tables which is posthoc.

During both the eccentric and concentric phase of the squat for $40 \% 1 \mathrm{RM}$ and $80 \% 1 \mathrm{RM}$, two lower body kinematic parameters were analysed. These parameters are comprised of knee valgus angle and maximum tibial rotation, for both the left and right knee. EMG data were also analysed for the five chosen muscles during the concentric phase of the squat for $40 \% 1 \mathrm{RM}$ and $80 \% 1 \mathrm{RM}$.

\section{RESULTS}

\section{Knee kinematics}

Table 1 shows the results for peak knee valgus angle and maximum tibial rotation value during both the $40 \%$ and $80 \%$ 1RM squats, while pairwise comparisons are displayed in table 2.

It should be noted that females had higher maximum tibial rotation means and lower peak knee valgus means compared with males across all conditions studied. This indicates that males may be at a higher risk of injury when squatting compared with females, regardless of whether a band is used or not. However, the effect that each resistance band had on knee kinematics during a squat was very similar between both males and females. Therefore, the results are discussed without referring to gender.

Peak knee valgus angle was highest in both the eccentric and concentric phases of the squat when an extra heavy band was used, and lowest when no band was used to squat. This was true for both the high intensity and low intensity squats. Similar findings were found in the left and right knee.

The extra heavy resistance band showed significantly higher peak knee valgus angles when compared with using no resistance band in the concentric phase (an increase of $28 \%$ was seen during the $80 \% 1 \mathrm{RM}$ squat and an increase of $37 \%$ was seen during the $40 \% 1 \mathrm{RM}$ squat) and the eccentric phase (an increase of $32 \%$ in peak knee valgus was seen during the $80 \% 1 \mathrm{RM}$ squat and an increase of $27 \%$ was seen during the $40 \% 1 \mathrm{RM}$ squat). The light resistance band also showed significantly greater peak knee valgus angles than the no resistance band condition in both the concentric ( $14 \%$ increase during the $80 \% 1 \mathrm{RM}$ squat and $27.2 \%$ increase during the $40 \% 1 \mathrm{RM}$ squat) and eccentric (19\% increase during the $80 \% 1 \mathrm{RM}$ squat and $18 \%$ during the $40 \% 1 \mathrm{RM}$ squat) phases of the squat.

Notable significant differences were recorded between the different squatting conditions with regard to tibial rotation. The extra heavy band resulted in the highest maximum tibial rotation value, while the no band condition resulted in the lowest value. One exception to this was in the left limb of the low intensity squat where the light resistance band condition elicited the highest maximum tibial rotation value.

The extra heavy band condition significantly increased maximum tibial rotation values by an average of $1.78^{\circ}$ compared with the no band condition during the $80 \%$
1RM squat. The same was true during the $40 \%$ 1RM squat where the extra heavy band produced an average increase in maximum tibial rotation of $1.75^{\circ}$. The pattern of results when comparing the light resistance band to the no band condition mirrored that of the extra heavy resistance band compared with the no band condition. The light band caused an increase in maximum tibial rotation of $1.55^{\circ}$ during the $80 \% 1 \mathrm{RM}$ squat and $1.93^{\circ}$ during the $40 \% 1 \mathrm{RM}$ squat, compared with the no band condition.

\section{Muscle activity}

Muscle activity results for the concentric phase will be studied as this phase of the squat generated the largest muscle activation values (figure 3 ). The extra heavy resistance band generated a significantly larger mean peak EMG value in GM than the light resistance band $80 \%$ 1RM left: $\mathrm{p}<0.001 ; 80 \% 1 \mathrm{RM}$ right: $\mathrm{p}<0.001 ; 40 \% 1 \mathrm{RM}$ left: $\mathrm{p}<0.001 ; 40 \% 1 \mathrm{RM}$ right: $\mathrm{p}<0.001)$ and the no band condition $(80 \%$ 1RM left: $\mathrm{p}=0.009 ; 80 \% 1 \mathrm{RM}$ right: $\mathrm{p}=0.005$; $40 \%$ 1RM left: $\mathrm{p}<0.001 ; 40 \%$ 1RM right: $\mathrm{p}<0.001)$. Quantifying this, the heaviest resistance band elicited a mean GM EMG value $13.25 \%$ higher than the no band condition during the high intensity squat and $23.15 \%$ higher during the low intensity squat. Significant difference was found in the activity of the right VL where the no band condition produced much higher activity than the extra heavy band (80\% 1RM: $\mathrm{p}=0.028)$ and the light band $(80 \% 1 \mathrm{RM}$ : $p=0.018 ; 40 \%$ 1RM: $p=0.005$ ). However, peak EMG value in the left VL during low intensity squats was significantly lower in the extra heavy band condition than no band $(\mathrm{p}=0.007)$ and the light band condition $(\mathrm{p}=0.031)$. During the low intensity squats for left VM, the no band condition also produced the highest peak EMG value and the extra heavy band was found to achieve significantly lesser muscle activity than both the no band condition $(\mathrm{p}=0.008)$ and the light band condition $(\mathrm{p}=0.008)$.

No other significant differences with regard to muscle activity were found.

\section{DISCUSSION}

The aim of this study was to determine whether looped resistance bands reduce peak knee valgus and internal tibial rotation, as well as their effect on lower body muscle activation during the barbell back squat. If resistance bands can successfully achieve what has been suggested then they will be beneficial in ACL rehabilitation situations, as stress on the ACL will be reduced. They will also be a useful piece of equipment for athletes as the resistance band could reduce the incidence of knee injuries within squatting, particularly injuries to the ACL, which are extremely debilitating to athletes. Hence two hypotheses to test: first, performing a barbell back squat with a resistance band will cause a decrease in peak knee valgus angle and a decrease in maximum tibial rotation angle. Second, performing a barbell back squat with a resistance band will cause an increase in muscle activation of all lower limb muscles tested. 


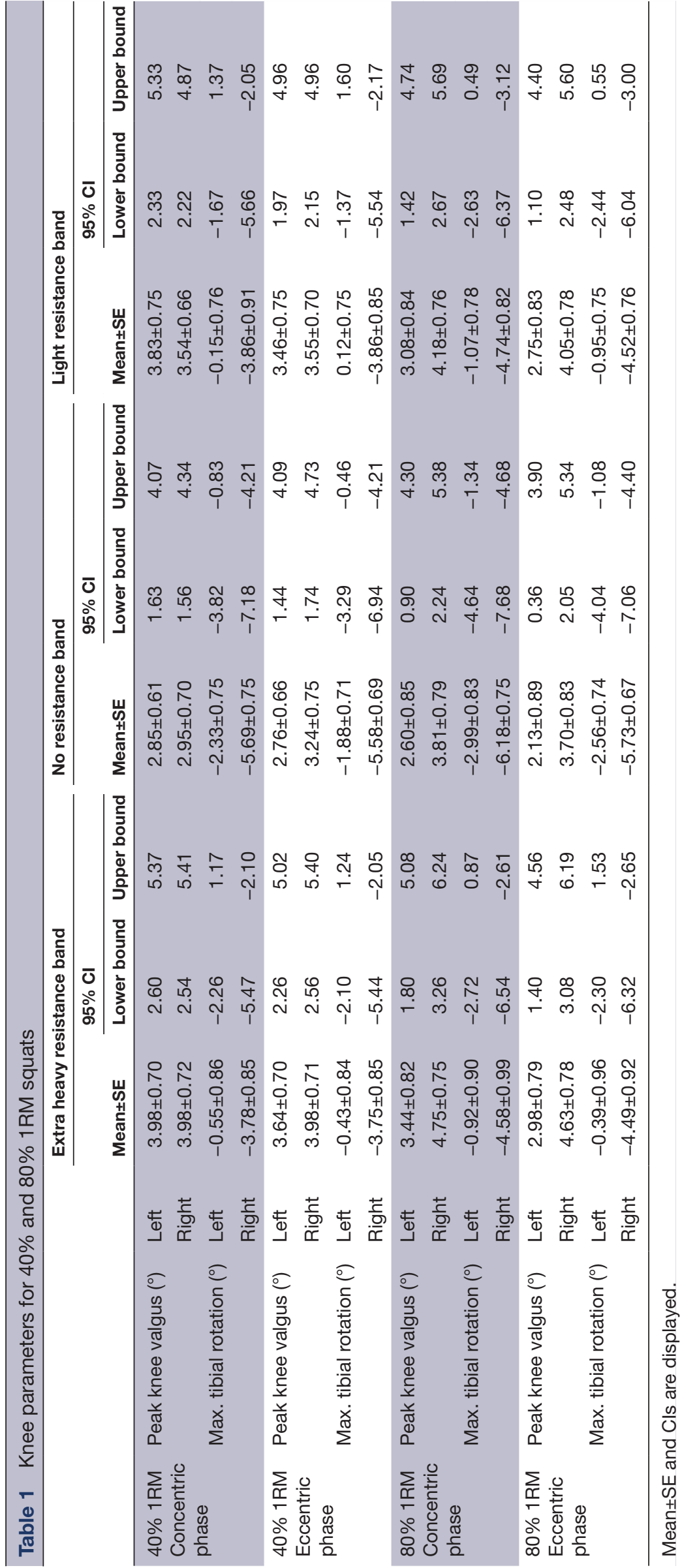


Table 2 Pairwise comparisons for 40\% and 80\% 1RM squats with $p$ values displayed

\begin{tabular}{|c|c|c|c|c|c|}
\hline & \multirow{2}{*}{\multicolumn{2}{|c|}{ Condition }} & \multirow{2}{*}{$\begin{array}{l}40 \% 1 \mathrm{RM} \\
\text { Squat } \\
\text { Sig. }\end{array}$} & \multirow{2}{*}{$\begin{array}{l}80 \% \text { 1RM } \\
\text { Squat } \\
\text { Sig. }\end{array}$} \\
\hline & & & & & \\
\hline \multicolumn{6}{|l|}{ Concentric phase } \\
\hline \multirow[t]{6}{*}{ Peak knee valgus $\left(^{\circ}\right)$} & Left & 1 & 2 & $0.000^{*}$ & $0.023^{*}$ \\
\hline & & 2 & 3 & $0.001^{*}$ & $0.045^{\star}$ \\
\hline & & 3 & 1 & 0.555 & 0.309 \\
\hline & Right & 1 & 2 & $0.000^{*}$ & $0.001^{*}$ \\
\hline & & 2 & 3 & $0.018^{*}$ & 0.169 \\
\hline & & 3 & 1 & 0.056 & $0.007^{*}$ \\
\hline \multirow[t]{6}{*}{ Max. tibial rotation $\left({ }^{\circ}\right)$} & Left & 1 & 2 & $0.000^{*}$ & $0.000^{*}$ \\
\hline & & 2 & 3 & $0.000^{*}$ & $0.000^{*}$ \\
\hline & & 3 & 1 & 0.286 & 0.747 \\
\hline & Right & 1 & 2 & $0.000^{*}$ & $0.002^{*}$ \\
\hline & & 2 & 3 & $0.000^{*}$ & $0.000^{\star}$ \\
\hline & & 3 & 1 & 0.829 & 0.624 \\
\hline \multicolumn{6}{|l|}{ Eccentric phase } \\
\hline \multirow[t]{6}{*}{ Peak knee valgus $\left(^{\circ}\right)$} & Left & 1 & 2 & $0.001^{*}$ & $0.026^{\star}$ \\
\hline & & 2 & 3 & $0.017^{*}$ & $0.033^{*}$ \\
\hline & & 3 & 1 & 0.521 & 0.495 \\
\hline & Right & 1 & 2 & $0.004^{*}$ & $0.001^{*}$ \\
\hline & & 2 & 3 & 0.160 & 0.156 \\
\hline & & 3 & 1 & 0.067 & $0.003^{*}$ \\
\hline \multirow[t]{6}{*}{ Max. tibial rotation $\left({ }^{\circ}\right)$} & Left & 1 & 2 & $0.001^{*}$ & $0.003^{*}$ \\
\hline & & 2 & 3 & $0.000^{\star}$ & $0.000^{*}$ \\
\hline & & 3 & 1 & 0.160 & 0.383 \\
\hline & Right & 1 & 2 & $0.001^{*}$ & $0.012^{\star}$ \\
\hline & & 2 & 3 & $0.000^{*}$ & $0.000^{*}$ \\
\hline & & 3 & 1 & 0.761 & 0.926 \\
\hline
\end{tabular}

1=Extra heavy resistance band. 2=No resistance band. 3=Light resistance band.

*Statistically significantly $(\mathrm{p}<0.05)$.

\section{Knee kinematics}

Results illustrate that in both phases of the squat, resistance bands increase peak knee valgus angle and internal tibial rotation in low intensity and high intensity barbell squats. This was true for both the light resistance band and the extra heavy resistance band. It must be noted that, although there was no significant difference between the light and extra heavy bands, the trend observed was that the higher the resistance the greater the two angles investigated. No other studies are known to have investigated these angles with regard to squatting with resistance bands. While Gooyers et al $(2012)^{3}$ and Foley et al $(2017)^{8}$ conducted similar investigations and used knee width index as the primary measure of medial knee collapse, results from both studies along with the current study support the notion that resistance bands do not promote neutral knee alignment during squatting. The current study expands on this by warning that performing the barbell back squat with a resistance band, regardless of strength, increases knee valgus.

A potential explanation for these results may be that, although the band could have acted as a proprioceptive aid and participants were continually encouraged to 'push their knees out against the resistance band', the strength of the resistance bands may have been too high for participants to overcome. This is a plausible assumption, as the majority of participants had never squatted with resistance bands before, meaning the hip abductor muscles may not be strong enough to resists the forces created by the band. This left participants with a peak knee valgus angle and tibial rotation value, in both the eccentric and concentric phases, higher than when no band was used. Therefore, resistance bands increase medial knee collapse which consequently raises the risk of injury to the knee. It would be interesting to see if the same results would be generated in individuals who have used resistance bands during the barbell squat exercise over a prolonged period of time, as they may have developed the necessary muscle activation patterns to overcome the forces produced.

\section{Muscle activity}

Gluteus maximus and gluteus medius

In agreement with previous literature ${ }^{89}$ the present study found that all participants experienced an overall significant increase in GM activity when using either the light resistance band or the extra heavy resistance band in the high intensity or low intensity squat, compared with the no band condition. Figure 4 illustrates raw EMG data of the GM muscle during the three squat conditions for one participant. The heavier band produced the largest increase, allowing us to conclude that, by increasing the strength of the resistance band, GM activity exponentially increases. This is in agreement with the results demonstrated by Spracklin et al (2017). ${ }^{9}$

This result can be explained as the forces produced by the resistance bands must be opposed by the hip abductor muscles, with the likely aim of resisting internal hip rotation. ${ }^{9}$ Therefore, individuals who aim to target the GM during squatting should achieve this goal through squatting with looped resistance bands.

Although the highest GM EMG value was achieved in the high intensity squat, it should be noted that a larger percentage increase in GM activity was achieved in the low intensity squat. Individuals who focus on low intensity squats could increase GM activation by a staggering $25 \%$ if a heavy resistance band is implemented.

The current study found no significant difference in the activity of the gluteus medius muscle with and without looped band intervention. This differs from the results found in previous research. ${ }^{89}$

\section{Biceps femoris}

There was no difference in the activity of $\mathrm{BF}$ when squatting with and without a resistance band, regardless of strength or squat intensity. This is in agreement with 

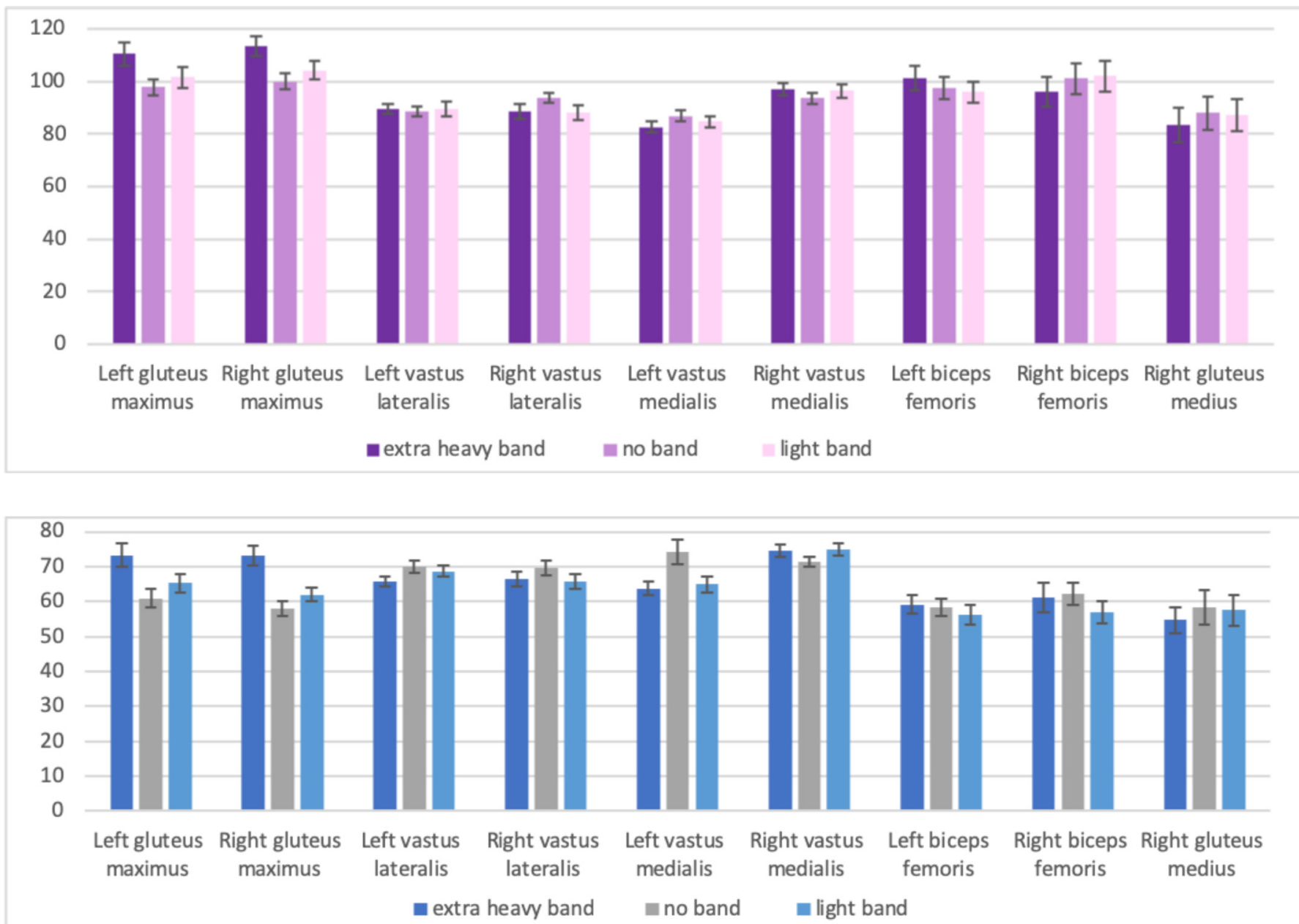

Figure 3 Mean peak EMG values as a \% of MVC during the concentric phase of $80 \% 1 \mathrm{RM}$ (top) and 40\% 1RM (bottom).

results from Spracklin et al (2017). ${ }^{9}$ The hamstrings are unlikely to contribute to resisting the forces produced by the resistance band, explaining the result.

Vastus lateralis and vastus medialis

Many individuals who squat aim to target the quadriceps; thus; any insight into how to gain increased muscle activity in the quadriceps is beneficial.

Although significant results were often not gained, the predominant trend was that quadriceps muscle activity was reduced when a resistance band was used to squat, compared with no band. This disputes the results found from previous research. ${ }^{8}$ The differing results may be attributed to the fact that squat depth was not controlled. However, the overall recommendations to squatters who focus on targeting the quadriceps rather than GM are consequently advised not to squat with resistance bands.

To summarise, the hypothesis that this study explored, "performing a barbell back squat with a resistance band will cause a decrease in peak knee valgus angle and a decrease in maximum tibial rotation', can be rejected. The hypothesis 'performing a barbell back squat with a resistance band will cause an increase in muscle activation of all lower limb muscles tested' can also be rejected as not all muscles were found to increase in activation.

\section{LIMITATIONS}

Squat depth was not controlled, meaning differing depths could have been achieved in the different conditions. This may have affected muscle activation results as the gluteus and quadricep muscles have been shown to increase in activation as squat depth increases. ${ }^{2} 14$

Despite apparent bilateral differences in the data collected, this study did not compare the left and right side as this was not included in the aim.

Although participants were experienced in practicing the exercise, one repetition maximums were self-reported and this could be considered a limitation. In addition, squat width was set as 'slightly wider than shoulder width' which was standardised between sets for each individual. However, foot width could have been calculated to ensure absolute standardisation between participants.

\section{CONCLUSION}

Squatting with resistance bands was shown to increase muscle activation of GM, while making no difference in the EMG amplitude of the hamstrings. However, overall results indicate that squatting with a resistance band reduces peak knee flexion angle, increases peak knee valgus angle and increases maximum tibial rotation 


\section{Gluteus Maximus}

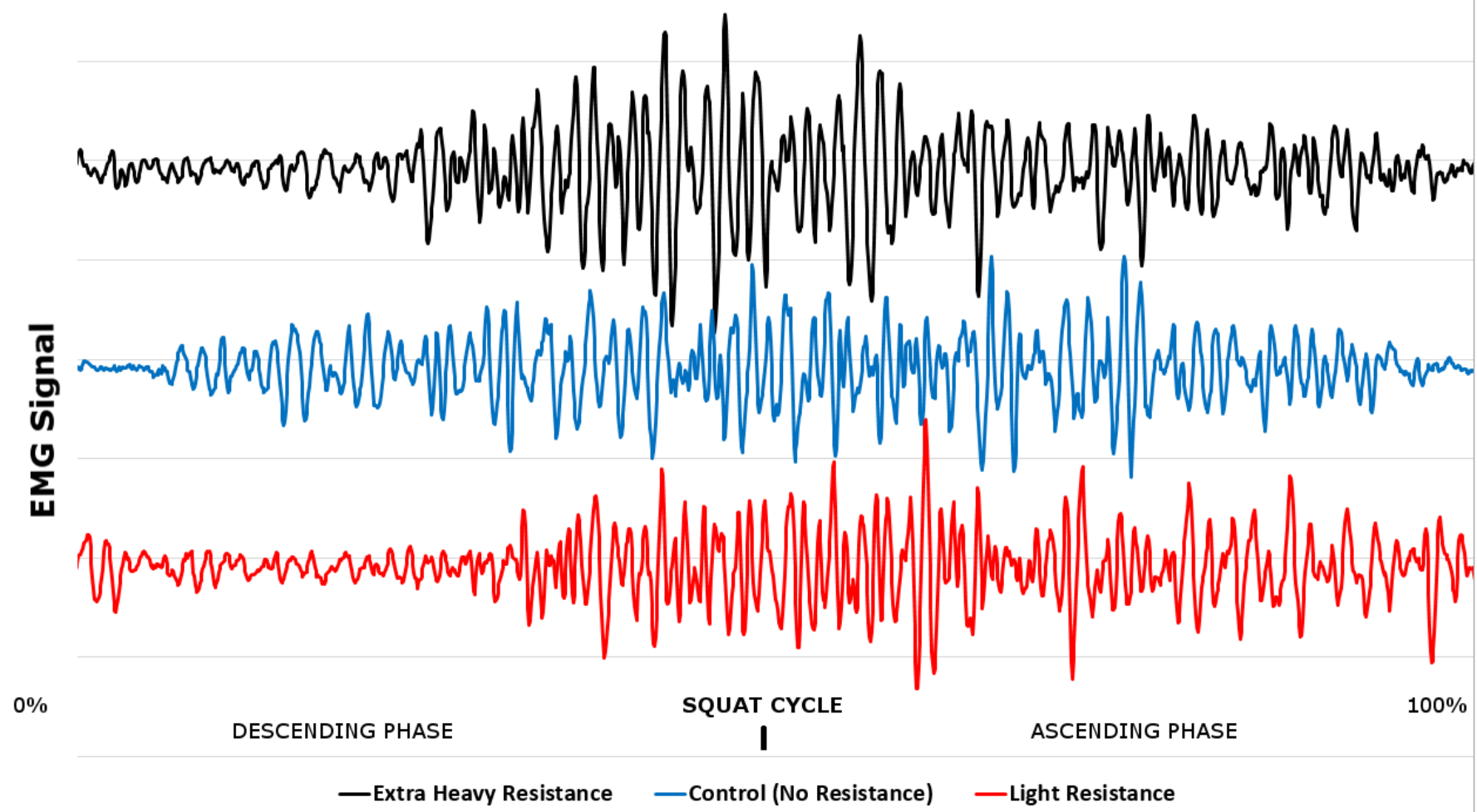

Figure 4 Raw EMG data of the GM muscle during the three squat conditions for one participant.

value. This may lead to an increased risk of knee injury during the barbell back squat.

Despite clinicians and coaches supporting the notion that resistance bands promote neutral knee alignment during squatting, the current study disagrees and advises consideration for the removal of this technique as it may lead to an increased risk of knee injury.

Although this study focused on the results being applicable to the population as a whole, future research could investigate if results differ between male and females. Long-term effects of the use of resistance bands during squatting is another area which could be explored.

\section{Acknowledgements The authors would like to thank Mr lan Christie for his} valuable assistance in the production of bespoke images.

Contributors All coauthors are in agreement to be accountable for all aspects of the work presented in this manuscript. MBR: planning the study, conducting the study, analysing the data, reporting the study, generating the draft write-up and responsible for the overall content as guarantor. GPA: data collection for study and Vicon markers repeatability. SN: data collection for study and Vicon software reliability. WWW: statistical analysis of data. RA: reporting the study, revising the original and revision manuscript critically for intellectual content, submitting the study and responsible for overall content as guarantor.

Funding The study was funded internally by the department.

Competing interests None declared.

Patient consent for publication Not required.

Ethics approval The study was approved by the Medical School Research Ethics Committee - ID SMED REC 098/18.

Provenance and peer review Not commissioned; externally peer reviewed.
Data availability statement No data are available. Data remains confidential until we publish all relevant information.

Open access This is an open access article distributed in accordance with the Creative Commons Attribution Non Commercial (CC BY-NC 4.0) license, which permits others to distribute, remix, adapt, build upon this work non-commercially, and license their derivative works on different terms, provided the original work is properly cited, appropriate credit is given, any changes made indicated, and the use is non-commercial. See: http://creativecommons.org/licenses/by-nc/4.0/.

ORCID iD

Rami Abboud http://orcid.org/0000-0002-1753-9606

\section{REFERENCES}

1 Escamilla RF, Fleisig GS, Zheng N, et al. Effects of technique variations on knee biomechanics during the squat and leg press. Med Sci Sports Exerc 2001;33:1552-66.

2 Schoenfeld BJ. Squatting kinematics and kinetics and their application to exercise performance. J Strength Cond Res 2010;24:3497-506

3 Gooyers CE, Beach TAC, Frost DM, et al. The influence of resistance bands on frontal plane knee mechanics during body-weight squat and vertical jump movements. Sports Biomech 2012;11:391-401.

4 Bell DR, Padua DA, Clark MA. Muscle strength and flexibility characteristics of people displaying excessive medial knee displacement. Arch Phys Med Rehabil 2008;89:1323-8. Elsevier.

5 Mauntel TC, Frank BS, Begalle RL, et al. Kinematic differences between those with and without medial knee displacement during a single-leg squat. J Appl Biomech 2014;30:707-12.

6 Bell DR, Oates DC, Clark MA, et al. Two- and 3-dimensional knee valgus are reduced after an exercise intervention in young adults with demonstrable valgus during squatting. J Athl Train 2013:48:442-9. National Athletic Trainers Association.

7 Padua DA, Bell DR, Clark MA. Neuromuscular characteristics of individuals displaying excessive medial knee displacement. $J$ Athl Train 2012;47:525-36. 
8 Foley RCA, Bulbrook BD, Button DC, et al. Effects of a band loop on lower extremity muscle activity and kinematics during the BARBELL squat. Int J Sports Phys Ther 2017;12:550-9.

9 Spracklin KF, Button D, Halperin I. Looped band placed around Thighs increases EMG of gluteal muscles without hindering performance during squatting. J Perform Heal Res 2017;1.

10 Hewett TE, Myer GD, Ford KR, et al. Biomechanical measures of neuromuscular control and valgus loading of the knee predict anterior cruciate ligament injury risk in female athletes: a prospective study. Am J Sports Med 2005;33:492-501.

11 COREZONE Sports. Resistance Loop Bands (6 Pack)-"Best resistance bands 2018" expertreviews [Internet], 2019. Available: https://www.corezonesports.co.uk/collections/resistance-bands/ products/resistance-loop-bands-6-pack [Accessed 11 Apr 2019].

12 Balshaw TG, Hunter AM. Evaluation of electromyography normalisation methods for the back squat. J Electromyogr Kinesiol 2012;22:308-19. Elsevier.

13 Baker D. The use of submaximal repetitions to predict maximal squat and bench press strength in trained athletes. Aust Strength Cond Assoc Mag 1995;3:17-19.

14 Caterisano A, Moss RF, Pellinger TK, et al. The effect of back squat depth on the EMG activity of 4 superficial hip and thigh muscles. $J$ Strength Cond Res 2002;16:428-32. 